\title{
The Effect of Bias Voltage and Ripple Voltage on Energy Loss in Aluminum Oxide Dielectric Films
}

\author{
Tomokazu HASEBE, ${ }^{a *}$ Robert S. ALWITT, ${ }^{\mathrm{b}}$ and Hidenori UCHI ${ }^{\mathrm{c}}$
}

\author{
${ }^{a}$ Chemi-Con Materials Corporation (366 Lexington Drive, Buffalo Grove, IL 60089, USA) \\ ${ }^{\mathrm{b} B o u n d a r y}$ Technologies, Inc. (P.O. Box 622, Northbrook, IL 60065-0622, USA) \\ 'Nippon Chemi-Con Corporation (363 Arakawa, Takahagi-shi, Ibaraki 318-8505, Japan)
}

Received December 4, 2000 ; Accepted March 1, 2001

\begin{abstract}
The energy loss in aluminum oxide dielectric films on etched capacitor foil was measured during application of bipolar constant current pulses. Pulse conditions produced a ripple voltage with approximate triangular waveshape superposed on a $d c$ bias voltage. The charge and discharge energies during a cycle were obtained by integration under the V-t trace, and the energy loss is expressed as the difference between these quantities divided by the charge energy. The energy loss increases with bias voltage and with ripple voltage. There are two factors contributing to the energy loss. One is a field-dependent oxide dissipation factor (DF), with either linear or quadratic dependence on bias voltage, depending on oxide thickness. The field dependence results from prior exposure of the oxide to elevated temperature. The other factor is a steady state absorption current that scales with the product of bias voltage and ripple voltage. Under conditions typical of capacitor applications the loss can be significantly greater than the usual small signal value of oxide DF measured with a LCR meter.
\end{abstract}

Key Words : Anodic Oxide, Dielectric Absorption, Dissipation Factor, Electrolytic Capacitor

\section{Introduction}

Aluminum electrolytic capacitors are widely used in power electronics applications, particularly inverter circuits for controlling the speed of ac motors. ${ }^{1,2)}$ Variable speed ac motors are used for traction drive in electric and hybrid vehicles, industrial motors for pumps and fans, and in home appliances. The capacitor sees a high $\mathrm{dc}$ bias voltage modulated by an ac voltage and this drives a large ripple current between the capacitor plates. The percent ripple voltage is of the order $1 \%$, and with $\mathrm{dc}$ bias as high as $300-450 \mathrm{~V}$, substantial energy flows through these capacitors. Energy loss in the capacitor during operation can cause significant temperature rise. $^{3,4}$ The largest contribution to loss is from ohmic heating due to the ripple current passing between the plates, through the electrolyte impregnated spacer. Dielectric loss also causes electrical energy to be degraded to heat within the oxide dielectric. This is usually considered to be a minor contribution, but our studies have shown that dielectric loss is greater than anticipated and can be significant under certain operating conditions.

The dissipation factor (DF) is the fraction of energy dissipated in a dielectric, $\mathrm{DF}=\varepsilon^{\prime \prime} \varepsilon^{\prime}$, where $\varepsilon^{\prime}$ and $\varepsilon^{\prime \prime}$ are the real and imaginary components of complex permittivity. The DF of anodic alumina is near 0.01 when measured at small bias and signal voltages, e.g., as with a LCR meter. We have reported some initial measurements of the energy loss of high voltage capacitor foils under conditions that simulate high ripple voltage service. ${ }^{5,6)}$ Energy loss in the oxide dielectric was found to increase with both bias voltage and ripple voltage and may be significantly larger than the small signal value.
There are two components to this energy loss: One is the contribution from oxide DF, and the other is a loss associated with the charge and discharge of the dielectric at ripple frequency.

It was recently reported that the field dependence of oxide DF depends on the oxide thermal history. ${ }^{7)}$ The dielectric properties of oxide grown at room temperature, and not subsequently exposed to a higher temperature, are not field dependent. Oxide exposed to elevated temperature exhibits field dependent $\mathrm{C}$ and $\mathrm{DF}$, and the field dependence increases with temperature. Commercial processes to deposit anodic oxide use hot formation baths, and it is common practice to anneal the formed foil at a temperature of $450^{\circ} \mathrm{C}$ or higher during its processing. As a result, commercial high voltage foils have field dependent dielectric properties. The cause of this field dependence is under investigation, and consideration of the phenomenon is beyond the scope of this paper.

In this paper we present comprehensive results of measurements of energy loss of high voltage anode foils. Voltage-time data are collected during application of a string of bipolar current pulses to a foil specimen. From these data the energy loss during a charge/discharge cycle is calculated. The dependence of energy loss on bias voltage, ripple voltage, frequency and temperature is reported.

\section{1 Foil Specimens}

\section{Experimental}

Capacitor foils are etched to enhance surface area, and then the oxide dielectric is deposited. The foils used in 
this study have a tunnel etch structure. ${ }^{8)}$ The oxide is either crystalline or amorphous, depending on selected formation process conditions, but the crystalline modification is most widely used. Three KDK brand foils manufactured by the Materials Division of Nippon Chemi-Con Corporation were used in this study. Two foils are coated with crystalline oxides, 560 and $590 \mathrm{~nm}$, respectively, and these have an increase in specific area by etching of about $20 \mathrm{x}$. One foil is coated with $560 \mathrm{~nm}$ of amorphous oxide and has area enhancement of about $10 \mathrm{x}$. Oxide formation is a continuous process and the end values of voltage and current, from which oxide thickness can be calculated, are not known precisely. Oxide thickness and field reported in this study is based on engineering estimates of final bath voltage and values of inverse field for amorphous and crystalline oxide of 1.4 and $0.95 \mathrm{~nm} \mathrm{~V}^{-1}$, respectively. ${ }^{8)}$ The calculated thickness is judged to be accurate to $\pm 5 \%$.

The foil specimen has a rectangular body, $25 \mathrm{~mm} \times 38$ $\mathrm{mm}$ or smaller, and a $6 \mathrm{~mm}$ wide handle extends along one edge. After cutting, the handle is masked and then healing of defects and formation of oxide on cut edges is done at $\sim 10 \mu \mathrm{A} \mathrm{cm} \mathrm{cm}^{-2}$ (specific area basis) in aqueous borate electrolyte at $85^{\circ} \mathrm{C}$. The voltage rises rapidly at this current and then abruptly stops rising, at which point the circuit is opened and the specimen discharged. Oxide thickness is estimated from the terminal voltage, $V_{\mathrm{t}}$.

\section{2 Test cell}

Most energy loss measurements were made at room temperature in an organic electrolyte consisting essentially of ammonium azeleate in ethylene glycol (resistivity $400 \mathrm{ohm} \mathrm{cm}$ ). A few measurements were made in aqueous ammonium borate electrolyte $(180 \mathrm{ohm} \mathrm{cm}, \mathrm{pH} 7.5)$. Steady state behavior was independent of electrolyte selection. The measuring cell was a one liter beaker in which a central anode foil specimen was surrounded by a cylinder of stainless steel mesh pressed against the beaker wall. The same results were obtained when an etched aluminum cathode foil was substituted for the stainless mesh.

\section{3 Electrical measurements}

After immersing the specimen in the test electrolyte the cell voltage was raised stepwise, over about $10 \mathrm{~s}$, to a constant voltage equal to the highest voltage the foil will be exposed to during cycling. This voltage was $80 \%$ of $V_{t}$, but no higher than $400 \mathrm{~V}$ for $V_{\mathrm{t}}>500 \mathrm{~V}$ because of voltage limitations of the bipolar current source. After 1-2 hr the current decayed to $<1 \mu \mathrm{A}$ and the voltage was removed and the cell discharged for about one hour. The first cycling sequence was then run. After completing a cycling sequence the specimen was shorted while the next set of operating conditions were entered into the computer, which took about 10 minutes. Then the next cycle sequence was started. If the foil specimen was to be used another day, it was rinsed, dried, stored in a dessicator and the next time it was again held at constant voltage and then discharged as described above. Replicate measurements made on the same day, and on different days, showed that this procedure produced re-

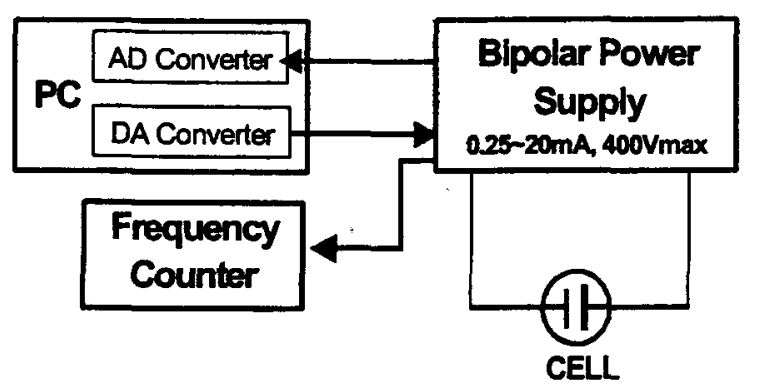

Fig. 1 Test circuit.

producible results, indicating that there were no changes in foil properties from test to test, and over extended time periods.

Figure 1 shows a diagram of the test circuit. A high voltage operational amplifier (Apex Microdevices model PA85) was configured as a bipolar current source. The applied current is typically $2-3 \mathrm{~mA}$, corresponding to a specific current density $\sim 10 \mu \mathrm{A} \mathrm{cm} \mathrm{cm}^{-2}$. Current was controlled to better than $\pm 0.1 \%$. Current polarity reversed at programmed voltage limits. The cell voltage is measured at $50 \mu$ s intervals, which compares with charge and discharge times $10^{3}$ greater. Voltage-time data were collected using a Keithley Instruments Metrabyte board, model DAS1600HR. We estimate accuracy of voltage readings to be better than $\pm 0.01 \%$. A single experiment consists of cycling between the preset voltage limits for 1,010 cycles, with collection of V-t data over 10 cycles starting at the 10,100 , and 1000 cycle points.

The constant current bipolar pulses produce approximately triangular V-t traces (Fig. 2a). As shown in Fig. $2 \mathrm{~b}$, the bias voltage is established by the charge time for the first pulse, and $\Delta V$ is set by the voltage limits for current reversal. Frequency depends on $\Delta V$, current density, and specimen capacitance, and is set by adjusting
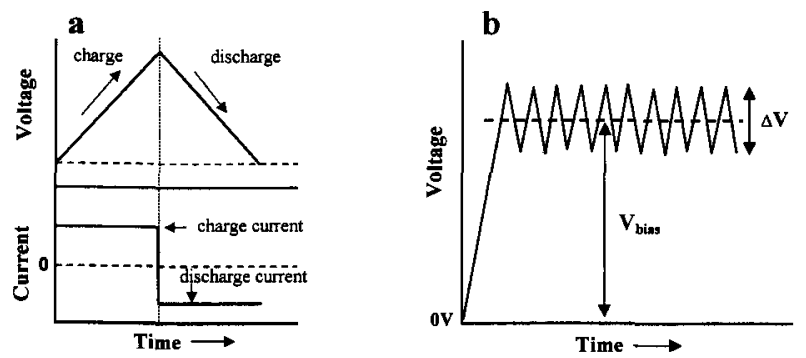

Fig. 2 Sketch of current pulses and V-t traces: (a) one cycle and (b) typical cycling.
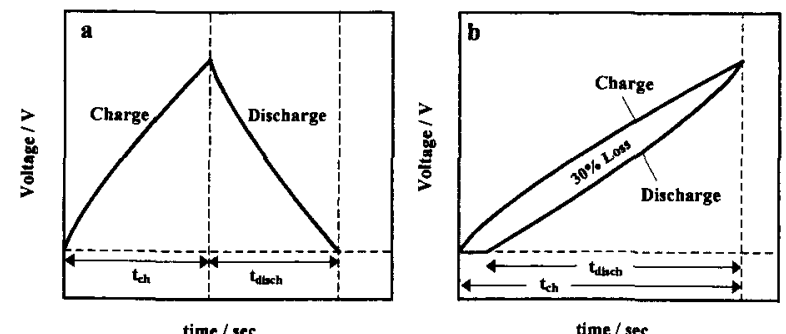

Fig. 3 Sketches of voltage-time response to constant current charge and discharge cycling for extreme case of $30 \%$ energy loss. 


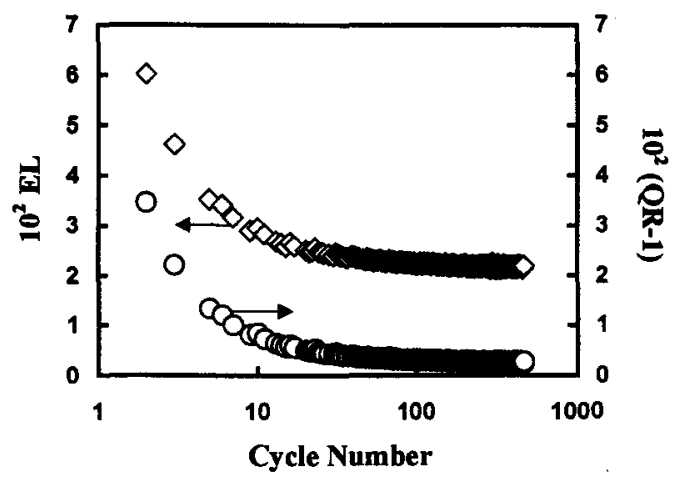

Fig. 4 Typical behavior of EL and $Q R-1$ during cycling.

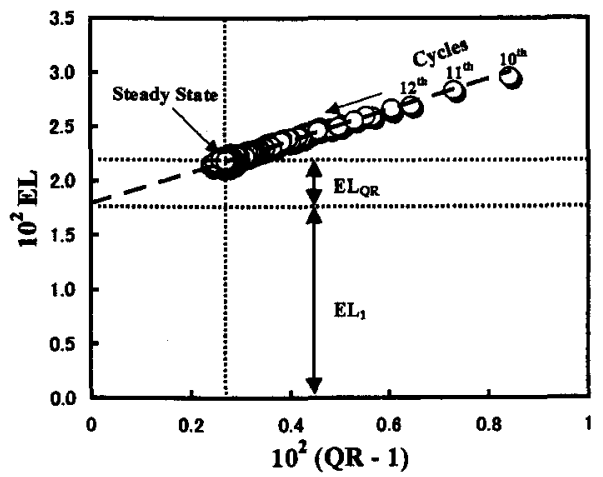

Fig. 5 Relation between EL and $Q R-1$ and definition of EL terms.

current at the start of the first run. Measurements were made at frequencies between 1 and $30 \mathrm{~Hz}$, with most measurements at $6 \mathrm{~Hz}$. A single experiment consisted of making a set of measurements with steps of increasing $V_{\text {bias, }}$ with other variables held constant. After measuring at the highest $V_{\text {bias }}$ usually an additional run at one of the lower $V_{\text {bias }}$ was done to confirm reproducibility.

The V-t traces are not linear; the voltage rise is slightly convex during charge, and slightly concave during discharge ${ }^{9)}$ A sketch of V-t trace with extreme curvature, to illustrate this behavior, is shown in Fig. 3a. In this example the loss is $30 \%$, about ten times the usual value. The area under a V-t charge curve, multiplied by the current, gives the charge energy, $J_{\mathrm{ch}}$. Similarly, the area under a V-t discharge curve multiplied by the current gives the discharge energy, $J_{\text {disch. }}$ The energy loss is expressed as a fraction of the charge energy, $\mathrm{EL}=\left(J_{\mathrm{ch}}-J_{\mathrm{dish}}\right) / J_{\mathrm{ch}}$. Thus, EL is the ratio of the area between the curves in Fig. $3 \mathrm{~b}$, where the discharge time has been folded back over the charge time scale, to the area under the charge curve between the voltage limits of $V_{\min }$ and $V_{\max }$. Discharge time is always shorter than charge time, with $t_{\text {disch }}$ $=t_{\text {ch }}$ approached as a limiting case as $\Delta V$ and $V_{\text {bias }} \rightarrow 0$.

The steady state losses are relatively small, in the range $0.4-4 \%$, so to get reliable and precise values requires careful treatment of the data. Area under a V-t trace was determined using the trapezoidal rule, with 1.5 $\mathrm{ms}$ subintervals, 30 data points/ subinterval. The average voltage for a subinterval was located at the average time of the subinterval, and the V-t trace was constructed by connecting these average points. In the vicinity of the polarity reversal $V$ was treated as increasing (or decreasing) at a constant rate. For example, one regression line was determined for the five subintervals before reversal at $V_{\max }$ and another line for a like number of subintervals after reversal. The intersection of these lines is the $\left(V_{\max }\right.$, $t)$ coordinate at reversal. For each cycle, error from any small offset in $V_{\min }$ at the end of discharge from the value at the start of charge was eliminated by interpolation or extrapolation of V-t near end of discharge to find $t_{\text {disch }}$ for $V=\left(V_{\text {min }}\right)_{\text {start }}$

\section{Results}

\section{1 General characteristics of the data}

Typical dependence of energy loss (EL) on cycle number is shown in Fig. 4. At the test frequency of $6 \mathrm{~Hz}$ the loss decreases to a nearly steady value after 100 cycles. In another experiment in which cycling was done for extended times, at 1 and $6 \mathrm{~Hz}$ a steady state was reached near $10^{3}$ cycles. At 19 and $28 \mathrm{~Hz} \mathrm{EL}$ continued to decrease between $10^{3}$ and $10^{4}$ cycles, by about $5 \%$. It was not practical to run for $10^{4}$ cycles, so $10^{3}$ cycles was taken as quasi-steady state.

With constant current operation the ratio of charge time to discharge time is equal to the charge ratio, $Q_{\mathrm{ch}} /$ $Q_{\text {disch }}$, which we call $Q$ ratio or $Q R$. For an ideal capacitor $Q R$ is 1 , but for real capacitors $Q R>1$ and its value approach 1 as a limiting case. $Q R$ and EL have similar dependency on cycle number, as seen in Fig. 4. It is found that after the 10th cycle $E L \propto Q R$, or $(Q R-1)$ as shown in Fig. 5. (The cycles decrease from upper right to lower left.) The $Q R$ and EL were calculated for each cycle, and the EL vs. $(Q R-1)$ regression line was determined from the individual values for cycles 10-19 and the average values for the 10 cycles at 100 and at $1000 \mathrm{cy}$ cles. The data point for 1000 cycles is $\left[E L_{s s},(Q R-1)_{\mathrm{ss}}\right]$, the quasi-steady state values. This usually fell on, or very close to, the regression line. If good agreement was not found between the line and this point, the data were rejected and the experiment repeated.

Extrapolation to $(Q R-1)=0$ gives the energy loss when charge and discharge times are equal, designated $E L_{1}$. The difference between $E L_{s s}$ and $E L_{1}$ is the loss associated with $Q R>1$, which we call $\mathrm{EL}_{Q R .}{ }^{* 1}$

$$
\mathrm{EL}=\mathrm{EL}_{1}+\mathrm{ELQR} \text {. }
$$

The difference between $Q_{\mathrm{ch}}$ and $Q_{\text {disch, }} \Delta Q$, can be expressed in terms of $Q R$,

$$
\Delta Q=Q_{\mathrm{ch}}(Q R-1) / Q R
$$

Since $Q R \approx 1,(Q R-1) / Q R \approx(Q R-1)$. Also, $Q_{\mathrm{ch}}=i_{\mathrm{ch}} /$ $2 f$, where $f$ is the cycle frequency. Substituting in eq. (2),

$$
\Delta Q=\left(i_{\mathrm{ch}} / 2 f\right)(Q R-1)
$$

${ }^{* 1}$ In a previous publication ${ }^{6)}$ what is here called $\mathrm{EL}_{\mathrm{QR}}$ was called ELic. The subscript stood for leakage current, but that terminology infers a particular interpretation of the data. 


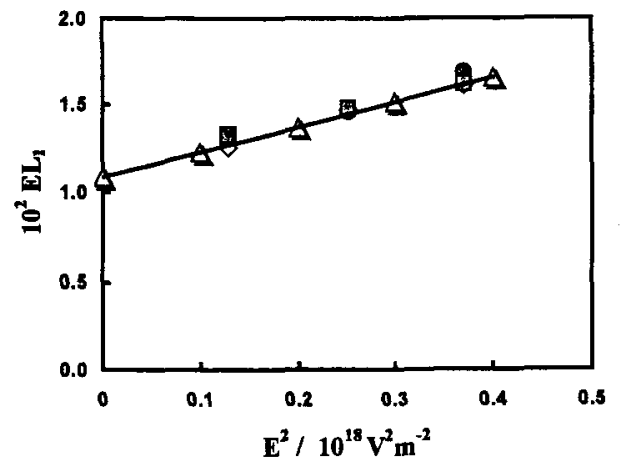

Fig. 6 Field dependence of EL for $560 \mathrm{~nm}$ crystalline oxide. Measurements at $6 \mathrm{~Hz}$ and $\Delta V$ of $(\bigcirc) 20 \mathrm{~V},(>) 40 \mathrm{~V}$, and (ם) $60 \mathrm{~V}$. Open triangles are oxide DF from impedance measurements. ${ }^{5}$ ?

If we express $\Delta Q$ as a loss current, $i_{q} / f$, then

$$
i_{\mathrm{q}} / i_{\mathrm{ch}}=(Q R-1) / 2
$$

The $i_{\mathrm{q}}$ term is the average loss current over the current pulse.

3. 2 Dependence of $E L_{1}, E L_{Q R}$ and $i_{q}$ on $V_{\text {bias, }}(V$, frequency and temperature

3. 2. 1 Properties of $\mathbf{E L}_{1}$ In Fig. 6 are $\mathrm{EL}_{1}$ results obtained with one foil, at several $V_{\text {bias }}$ and $\Delta V$ but at one frequency and temperature. Several important properties are illustrated. There is a regular increase in $\mathrm{EL}_{1}$ with average field, E. For the specimen in Fig. 6, EL has a quadratic dependence on field, and at the highest field is $60 \%$ larger than the zero bias value. (The highest field in these tests is close to the operating condition in a capacitor.) $E L_{1}$ is not dependent on $\Delta V$. Finally, oxide DF, calculated from impedance measurements (open triangles) is identical with $\mathrm{EL}_{1}$. Impedance was measured using a Solartron 1250 frequency response analyzer with a model 1296 Dielectric Interface that allows measurement at high bias voltage and small signal voltage over a wide frequency range. That work is reported elsewhere. ${ }^{7)}$ From those impedance measurements we summarize these properties of oxide DF (and $\mathrm{EL}_{1}$ ):

1. The field dependence is either linear or quadratic, depending only on oxide thickness. Oxide thicker than $500 \mathrm{~nm}$ has quadratic dependence (as in Fig. 6), whereas thinner oxide has a linear dependence.

2. Both amorphous and crystalline oxides exhibit field dependence, and for both there is a change in functional dependence from linear to quadratic at about $500 \mathrm{~nm}$ thickness.

3. At a field equal to the operating condition in a capacitor, the oxide DF at $25 \mathrm{C}$ is typically $40-60 \%$ larger than the value measured at zero bias, as with a LCR meter.

4. Oxide DF decreases linearly with $\log \mathrm{f}$ by $10 \%$ per decade, from about 3 to $300 \mathrm{~Hz}$. The frequency dependence increases with field but the change is small.

In addition, it is found that $\mathrm{EL}_{1}$ is independent of temperature up to $85 \mathrm{C}$, the highest test temperature.
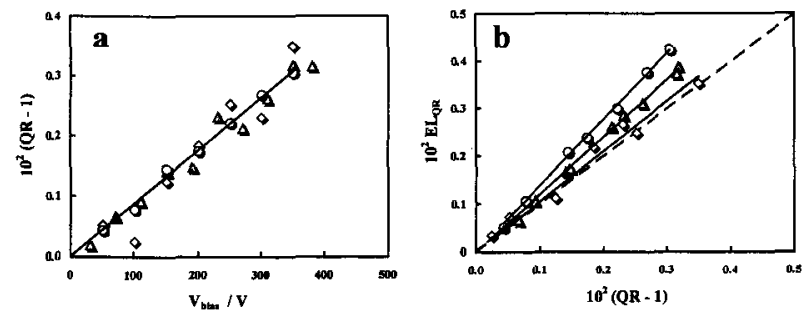

Fig. 7 Dependence of $Q R-1$ and ELQR on bias voltage and $\Delta V$ for $590 \mathrm{~nm}$ crystalline oxide at room temperature, freq. $=$ $6.0 \mathrm{~Hz}$. (a) $Q R-1$ vs bias voltage and (b) $\mathrm{EL}_{\mathrm{QR}}$ vs $Q R-1 . \Delta V$ : $(\diamond) 20 \mathrm{~V},(\boldsymbol{A}) 40 \mathrm{~V},(\mathrm{O}) 80 \mathrm{~V}$.

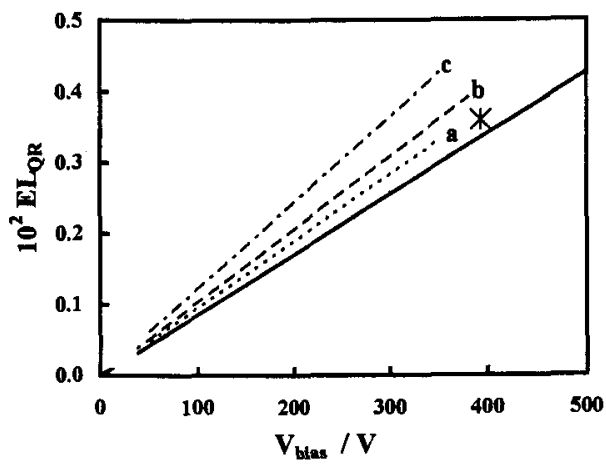

Fig. 8 Dependence of ELQR on bias voltage. $\Delta V$ : (a) $20 \mathrm{~V}$, (b) $40 \mathrm{~V}$, (c) $80 \mathrm{~V}$. Constructed from data in Fig. 7.

3. 2. 2 Properties of $\mathrm{EL}_{Q R}$ Figure 7a shows steady state values for $(Q R-1)$ obtained at different $V_{\text {bias }}$ and $\Delta V$, and indicates that $(Q R-1)_{\mathrm{ss}}$ is proportional to $V_{\text {bias }}$ and independent of $\Delta V$. Figure $7 \mathrm{~b}$ is data from the same experiment and shows that $\operatorname{ELQR}_{Q R} \propto(Q R-1)$, and hence $\mathrm{EL}_{\mathrm{QR}} \propto V_{\text {bias. }}$. There is some scatter to the $\Delta V=20$ $\mathrm{V}$ data, but that regression line lies slightly above the dashed line which represents $\mathrm{EL}_{Q R}=(Q R-1)$. In our experience, we always find $\mathrm{EL}_{Q R} \geq(Q R-1)$ and the dashed line represents the limiting case.

Figure 8 is constructed from Figs. $7 \mathrm{a}$ and $7 \mathrm{~b}$, with the solid line in Fig. 8 replacing the dashed line in Fig. $7 \mathrm{~b}$. For triangular waveshape,

$$
\% \text { ripple }=\left[\Delta V /(2 \sqrt{ } 3) V_{\text {bias }}\right] 100
$$

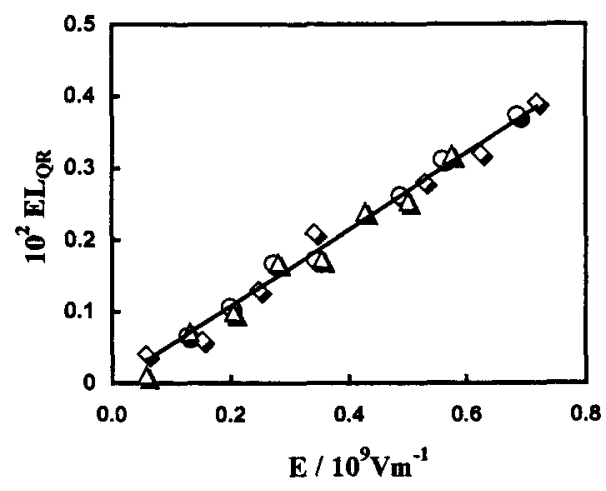

Fig. 9 Field dependence of ELQR for three capacitor foils: $(\diamond) 560 \mathrm{~nm}$ crystalline, (O) $590 \mathrm{~nm}$ crystalline, and (A) 560 $\mathrm{nm}$ amorphous. Measured at $6.0 \mathrm{~Hz}, \Delta \boldsymbol{V}=40 \mathrm{~V}$, and room temperature. 
The cross in Fig. 8 marks a condition of $1 \%$ ripple voltage. In the vicinity of the cross mark, changing $\Delta V$ by a factor of two would cause only small change in $\mathrm{EL}_{Q R}$. It appears that at ripple conditions likely to obtain in a capacitor there is only a small effect from $\Delta V$ on $\mathrm{EL}_{\mathrm{QR}}$.

Figure 9 shows the field dependence of ELQR is the same for the two crystalline oxides and the amorphous oxide. Of course, for the same formation voltage, amorphous oxide is $40 \%$ thicker than crystalline oxide, so with amorphous oxide the operating field in a capacitor is about $70 \%$ of the value for crystalline oxide, and ELQR is accordingly smaller. Note that for this thickness of oxide $E L_{1} \propto E^{2}$, but $E L_{Q R} \propto E$.

$E L_{Q R}$ does not change with frequency, between 1 and $30 \mathrm{~Hz}$. Only a few measurements were made at higher temperature of 55 and $85^{\circ} \mathrm{C}$, and those data were more scattered than at room temperature. It appeared that ELQR increases slowly with temperature in this range. It should be noted that always it was found that ELQR was less than $\mathrm{EL}_{1}$, but large enough to make a significant contribution to total EL.

\section{1 Characteristics of $i_{9}$}

\section{Discussion}

Eq. 4 shows that the current ratio, $i_{\mathrm{q}} / i_{\mathrm{ch}}$, is proportional to $(Q R-1)$. Then Fig. 7a is also a representation of the voltage dependence of the current ratio. The experimental design requires $i_{\mathrm{ch}} \propto \Delta V$ at fixed frequency, so from the linear relation in Fig. $7 \mathrm{a}$,

$$
i_{\mathrm{q}}=a V_{\text {bias }} \Delta V \text {. }
$$

Because $V_{\text {bias }}=\left(V_{\max }+V_{\min }\right) / 2$ and $\Delta V=\left(V_{\max }-V_{\min }\right)$, it follows that

$$
i_{\mathrm{q}}=(a / 2)\left(V_{\max ^{2}}{ }^{2} V_{\min }^{2}\right) .
$$

An important question is with regard to the nature of $i_{q}$ : Is it a leakage current or $a$ dielectric absorption current? There can be no ionic conduction through the oxide at the fields used in these experiments, and electronic leakage current has an exponential dependence on field, e.g., $i_{\mathrm{el}} \propto \exp (\sqrt{ } \mathrm{E})$, and such dependence was never seen. Depending on how one interprets Eqs. 6 and 7 , the current exhibits a linear or quadratic dependence on voltage (or field), and Fig. 7a shows the relation holds even at the lowest voltages.

When a voltage step is impressed on $\mathrm{Al}$ or $\mathrm{Ta}$ anodic oxide film, after initial charging, the current decays as $i$ $\propto V \mathrm{t}^{-\mathrm{n}}$, with $\mathrm{n}$ usually between 0.8 and 1.0. This is the dielectric absorption current arising from change in dielectric polarization, e.g., dipole reorientation. ${ }^{10,11)}$ Young and coworkers ${ }^{11)}$ found this relation to hold over $3 \mathrm{dec}-$ ades of time for anodized sputtered Ta films. At room temperature and moderate fields this relation was found for Al electrolytic capacitors and Ta solid capacitors from $<10 \mathrm{~s}$ to near $10^{4} \mathrm{~s}$, when the measurement was stopped. ${ }^{12)}$ The large time scale, and hence wide range of relaxation times for polarization processes, is associated with the amorphous or poorly crystalline state of the ox-

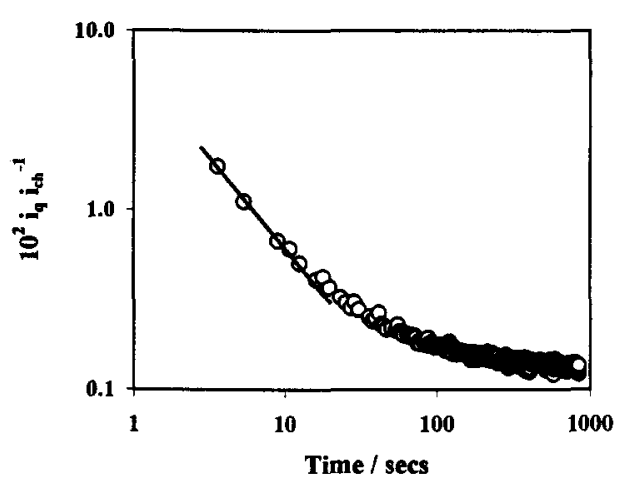

Fig. 10 Logarithmic plot of $i_{\mathrm{q}} / i_{\text {ch }}$ vs cycle number for 560 $\mathrm{nm}$ crystalline oxide. Test conditions are $V_{\text {avg }}=330 \mathrm{~V}, \Delta V=$ $60 \mathrm{~V}, 0.56 \mathrm{~Hz}$, room temperature.

\section{ide dielectric.}

According to linear response theory the real and imaginary parts of permittivity are linked to the voltage step response, i.e., the time dependent absorption current. This calculation can be completed only for certain limited conditions. Young and coworkers ${ }^{11)}$ calculated $\varepsilon^{\prime \prime}$ at particular (low) frequencies from the values of absorption current at selected times, and these results agreed with extrapolated ac bridge measurements. This confirmed the previous analysis by Baird ${ }^{13)}$ relating the dielectric loss and the absorption current.

Under ripple conditions Eq. 6 shows $i_{\mathrm{q}}$ ( $V_{\text {bias }}$ but enhanced by $\Delta V$. Figure 10 is a logarithmic plot of $i_{\mathrm{q}} / i_{\mathrm{ch}}$ vs. time on ripple load for an experiment run at $0.56 \mathrm{~Hz}$. Since $i_{\mathrm{ch}}$ is constant, the change in $i_{\mathrm{q}} / i_{\mathrm{ch}}$ depends only on $i_{\mathrm{q}}$. Initially, $i_{\mathrm{q}}$ decays as $\mathrm{t}^{-1}$ but only for about $\sim 20 \mathrm{~s}(10$ cycles). Then the decay rate slows and $i_{q}$ reaches a quasi-steady value after cycling for about $10^{3} \mathrm{~s}$. At the shortest times, the absorption current due to polarization by $V_{\text {bias }}$ is dominant, but the voltage modulation interacts with relaxation processes with time constants of the order of the ripple period or longer, and a steady state current, $i_{\mathrm{q}}$, is established. We propose that $i_{\mathrm{q}}$ is an absorption current, in that it is the system response to dielectric polarization, but we call it a ripple absorption current to identify its origin. The ripple absorption current results in $\mathrm{EL}_{Q R}$.

\section{Conclusions}

The fractional energy loss in aluminum oxide dielectric films on commercial capacitor foils increases with bias voltage and with ripple voltage. This is true for both crystalline and amorphous anodic oxides. Under conditions typical of capacitor applications the loss can be significantly greater than the usual small signal value of oxide DF measured with a LCR meter. There are two factors contributing to the energy loss. One is a fielddependent oxide DF, with either linear or quadratic dependence on bias voltage, depending on oxide thickness. The field dependence results from prior exposure of the oxide to elevated temperature. The other factor is a steady state absorption current that scales with the product of bias voltage and ripple voltage. 


\section{Acknowledgement}

We acknowledge the contribution of Mr. Scott Stillwell for design and construction of the high voltage bipolar current source, and for writing much of the software used in data acquisition and analysis.

\section{References}

1) W. Leonhard, Proc. IEEE, 76, 456 (1988).

2) D. J. BenDaniel and E. E. David, Jr., Science, 206, 773 (1979).

3) M. D. Weaver, 18th Capacitor and Resistor Technology Symposium, p.6, The Components Technology Institute, Huntsville, AL (1998).

4) J. L. Stevens, J. D. Sauer, and J. S. Shaffer, 15th Capacitor and Resistor Technology Symposium, p.56, The Components Technology Institute, Huntsville, AL (1995).

5) R. S. Alwitt and T. Hasebe, 18th Capacitor and Resistor Technology Symposium, p.149, The Components Technol- ogy Institute, Huntsville, AL (1998).

6) R. S. Alwitt and T. Hasebe, Electrolytic Condenser Rev., 50, 18 (1999) [publication of EIAJ, Japan].

7) R. S. Alwitt and T. Hasebe, Oxide Films (Eds. K. R. Hebert, R. S. Lillard and B. R. MacDougall), PV 2000-4, p. 283, The Electrochemical Society Proceedings Series, Pennington, NJ (2000).

8) H. Uchi, T. Kanno, and R. S. Alwitt, J. Electrochem. Soc., 148, B17 (2001).

9) R. S. Alwitt, presented at the IEEE Industry Applications Society Meeting, New Orleans, Oct, 5-9 (1997).

10) F. Argall and A. K. Jonscher, Thin Solid Films, 2, 185 (1968).

11) D. L. Pulfrey, P. S. Wilcox, and L. Young, J. Appl. Phys., 40, 3891 (1969).

12) R. S. Alwitt, unpublished results.

13) M. E. Baird, Rev. Modern Phys., 40, 219 (1968). 